\title{
Failure to Protect Yellowtail Seriola quinqueradiata against Nocardiosis by Vaccination with a Recombinant Protein and pcDNA4 Expression Vector of Edwardsiella tarda Glyceraldehyde-3-phosphate Dehydrogenase
}

\author{
Masayuki Imajoh ${ }^{1 *}$, Kazuki Tamura ${ }^{1}$, Kazuki Oguro ${ }^{1}$, Sumire Kurihara ${ }^{1}$, Jin Yamane ${ }^{1}$, Masato \\ Shimizu ${ }^{1}$, Syun-ichirou Oshima ${ }^{2}$ and Kenji Kawai ${ }^{3}$ \\ ${ }^{1}$ Laboratory of Fish Disease, Faculty of Agriculture, Kochi University, Kochi 783-8502, Japan \\ ${ }^{2}$ Graduate School of Kuroshio Science, Kochi University, Kochi 783-8502, Japan \\ ${ }^{3}$ The United Graduate School of Agricultural Sciences, Ehime University, \\ Ehime 790-8566, Japan
}

(Received December 16, 2013)

\begin{abstract}
Vaccination with glyceraldehyde-3-phosphate dehydrogenase (GAPDH) of Edwardsiella tarda has been demonstrated to have cross-protection against some other bacterial pathogens. We prepared a recombinant protein and pcDNA4 expression vector of GAPDH of $E$. tarda and injected those to 0 -age yellowtail Seriola quinqueradiata to evaluate their protective efficacies against Nocardia seriolae. The 60 -d survival rates ranged from $0 \%$ to $6.7 \%$ among vaccinated and control groups, suggesting that the protein and expression vector of $E$. tarda GAPDH had no protective effect against nocardiosis. However, the copy numbers of the $16 \mathrm{~S}$ ribosomal RNA gene of $N$. seriolae were significantly lower in the gill, kidney, and spleen of vaccinated fish 9 and 18 days post-infection compared with those in the control group that was also challenged with $N$. seriolae. Additionally, the CC chemokine, major histocompatibility complex class // $\alpha$ antigen, interleukin $1 \beta$, and immunoglobulin $M$ genes were significantly upregulated in the vaccinated groups. Four predicted B-cell epitopes of GAPDH in E. tarda, which may play an important role in cross-protection, differed in amino acid sequences from those of GAPDH in N. seriolae. This may explain the lack of a protective effect of $E$. tarda GAPDH against $N$. seriolae.
\end{abstract}

Key words: Edwardsiella tarda, Nocardia seriolae, GAPDH, vaccine, adjuvant, yellowtail, B-cell epitope

Nocardia are aerobic, gram-positive, non-motile, filamentous actinomycetes. Nocardia seriolae belongs to the genus Nocardia, and causes nocardiosis in fish (Kudo et al., 1988). In Japan, the disease is a major problem in cultured yellowtail Seriola quinqueradiata, amberjack Seriola dumerili, striped jack Pseudocaranx dentex, and olive flounder Paralichthys olivaceus (Takahashi et al., 2011).

There is currently no licensed vaccine for $N$. seriolae. Several studies have attempted to develop formalin-killed, heat-killed, and live attenuated vaccines of $N$. seriolae (Kusuda and Nakagawa, 1978; Kusuda et al., 1989; Shimahara et al., 2005; Itano et al., 2006). The formalin-killed vaccine did not have a protective effect against $N$. seriolae in vaccinated yellowtail, in which anti- $N$. seriolae antibody titer increased (Shimahara et al., 2005). Live attenuated vaccine suc-

\footnotetext{
* Corresponding author

E-mail: m-imajoh@kochi-u.ac.jp
}

cessfully protected yellowtail from lethal challenge by $N$. seriolae, but its long-term safety remains unclear (Itano et al., 2006). Recently, Kato et al. (2012) showed that Mycobacterium bovis bacillus Calmette-Guérin vaccine had the same protective properties as the live attenuated vaccine.

A $37-\mathrm{kDa}$ outer membrane protein (OMP) of Edwardsiella tarda is conserved among the different serotypes, and is a good vaccine candidate against edwardsiellosis in olive flounder (Kawai et al., 2004). The 37-kDa OMP is homologous to glyceraldehyde-3phosphate dehydrogenase (GAPDH), which is a cytoplasmic enzyme common in organisms (Liu et al., 2005). Our previous study showed that recombinant GAPDH (rGAPDH) protein of $E$. tarda expressed in Escherichia coli conferred definitive protection on olive flounder against vibriosis caused by Vibrio anguillarum (Liu et al., 2007). Liang et al. (2012) reported that immunoglobulin M (IgM) antibodies induced by rGAPDH of E. tarda exhibited obvious cross-reactions with $V$. 
anguillarum, Vibrio alginolyticus, Vibrio harveyi, and Aeromonas hydrophila. We postulated that GAPDH of E. tarda may be a multipurpose vaccine candidate against different pathogenic bacteria. Moreover, GAPDH of $E$. tarda can be used as a potential adjuvant for subunit vaccine of red sea bream iridovirus (Shimmoto et al., 2010).

In this study, we evaluated the protective efficacy of GAPDH of $E$. tarda against nocardiosis in yellowtail.

\section{Materials and Methods}

\section{Bacterial strain and culture conditions}

N. seriolae strain N-2927 was originally isolated from the spleen of cultured yellowtail in Kochi Prefecture, Japan, in 2007. The strain was picked from glycerol stock, and grown on a tryptic soy broth (TSB; Difco) plate for 5 days at $28^{\circ} \mathrm{C}$. A colony from the TSB plate was picked, and pre-cultured in $20 \mathrm{~mL}$ of TSB medium in a shaking incubator for 4 days at $28^{\circ} \mathrm{C}$. A bacterial suspension (100 $\mu \mathrm{L}$ ) was inoculated into $300 \mathrm{~mL}$ of TSB medium, and cultured by shaking incubation at $28^{\circ} \mathrm{C}$ for 4 days.

Construction, expression, and purification of rGAPDH protein

The cold shock expression vector pColdl (TaKaRa) was used to express the rGAPDH protein of $E$. tarda strain EF-1, which was originally isolated in 1979 from cultured Japanese eel Anguilla japonica in Shizuoka Prefecture, Japan (Kusuda et al., 1988), in E. coli. A 1,006-bp fragment including the full-length GAPDH gene, and the Ndel and $E c o R I$ sites was amplified by polymerase chain reaction (PCR) with the following primer set: forward primer, AACATATGACTATCAAAGTAGGTATTAACG; and reverse primer, AAGAATTCCTTAGAGATGTGAGCGATTA (the restriction enzyme recognition sites are underlined). PCR was performed using Ex Taq polymerase (TaKaRa). After digestion with $N d e l$ and $E c o R I$, the amplified product was purified from $1.0 \%$ agarose gel using a High Pure PCR product purification kit (Roche). The fragment was cloned into the $\mathrm{Ndel} / \mathrm{EcoRI}$ sites of the pColdl vector, which was transformed into E. coli strain BL21 (DE3). Overproduction of the soluble rGAPDH protein was induced by adding $100 \mathrm{~mm} \mathrm{IPTG}$ and incubated overnight at $15^{\circ} \mathrm{C}$. The recombinant protein was purified using complete his-tag purification resin (Roche), and dialyzed overnight at $4^{\circ} \mathrm{C}$ in dialysis buffer consisting of phosphate buffered saline (PBS) containing $2 \mathrm{~mm}$ ethylenediaminetetraacetic acid (EDTA) and $1 \mathrm{~mm}$ dithiothreitol. Protein concentration was measured using a Quant iT protein assay kit (Invitrogen) on a Qubit fluorometer (Invitrogen).
Construction, extraction, and purification of GAPDHexpression vector

The eukaryotic expression vector pcDNA4/V5-His (Invitrogen) was selected. A 1,026-bp fragment including the full-length GAPDH gene and the EcoRV and Xhol sites was amplified by PCR with the following primer set: forward primer, TAAGATATCATGGGAGCAACTATAATGACTATCAAAGTAG; and reverse primer, AAACTCGAGCTTAGAGATGTGAGCGATTAGGTCTAG (the restriction enzyme recognition sites are underlined). The digested, purified PCR product was cloned into the EcoRV/Xhol sites of the pcDNA4/V5-His vector, and transformed into E. coli strain JM109. The vector was purified on a large scale using a Genopure plasmid maxi kit (Roche). The pelleted pcDNA4-GAPDH (pGAPDH) vector was resuspended in TE buffer (10 mM Tris- $\mathrm{HCl}, \mathrm{pH}$ 8.0, and $1 \mathrm{~mm}$ EDTA), and was stored at $-20^{\circ} \mathrm{C}$ until use.

\section{Preparation of formalin-killed vaccine}

Formalin-killed $N$. seriolae cells (FKC) were prepared as described previously (Kusuda et al., 1989).

\section{Fish}

Zero-year-old yellowtail were caught in the Tosa Bay area, Japan. The fish were held in a 2-kL circular fiberglass reinforced plastic (FRP) tank with a continuous flow of sand-filtered sea-water, and were hand fed a commercial dry pellet diet once daily (Marubeni Nissin Feed) corresponding to approximately $3 \%$ of the fish body weight.

\section{Administration of $p G A P D H$ vector and $r G A P D H$ protein}

Yellowtail weighing about $26.6 \mathrm{~g}$ were divided into the following groups: pGAPDH-administered group, 54 fish were injected intramuscularly with the PGAPDH vector $\left(200 \mu \mathrm{g} \mathrm{fish}^{-1}\right)$; rGAPDH-administered group, 39 fish were injected intraperitoneally with the rGAPDH protein $\left(340 \mu \mathrm{g} \mathrm{fish}^{-1}\right.$ ); FKC-administered group, 39 fish were injected intraperitoneally with FKC $\left(8.0 \times 10^{5}\right.$ colonyforming units (CFU) fish ${ }^{-1}$ ); FKC- and pGAPDH-administered group, 33 fish were injected intraperitoneally with FKC $\left(4.0 \times 10^{5} \mathrm{CFU} \mathrm{fish}^{-1}\right)$ and intramuscularly with the pGAPDH vector $\left(200 \mu \mathrm{g} \mathrm{fish}^{-1}\right)$; and FKC- and rGAPDH-administered group, 33 fish were injected intraperitoneally with FKC $\left(4.0 \times 10^{5} \mathrm{CFU}\right.$ fish $\left.^{-1}\right)$ and intraperitoneally with the rGAPDH protein $(340 \mu \mathrm{g}$ $\left.\mathrm{fish}^{-1}\right)$. Sixty-two fish were injected with PBS as nonadministered groups.

\section{Challenge experiment}

The bacterial suspension was added to $10 \mathrm{~L}$ of seawater at a concentration of $7.5 \times 10^{5} \mathrm{CFU} \mathrm{mL}^{-1}$. At 28 days after administration, all administered and 37 nonadministered fish were immersed in sea-water for 10 min, and each group was immediately placed into a 
separate $200-\mathrm{L}$ tank. Water temperature in the tanks was $27.5^{\circ} \mathrm{C}-29.0^{\circ} \mathrm{C}$ during the challenge period. The remaining 25 non-administered fish were immersed in $10 \mathrm{~L}$ of sea-water mixed with $50 \mathrm{~mL}$ of sterile TSB medium. One fish in each group was sampled randomly at $1,3,5,7,9,14,18,36$, and 60 days post-infection (p.i.).

\section{Expression analysis of immune-related genes}

Total RNA was extracted from the liver and spleen tissue samples at $1,3,5,7,14,36$, and 60 days p.i. using Sepasol RNA I super G (Nakalai Tesque). Total RNA (1 mg) was transcribed to complementary DNA using ReverTraAce qPCR RT master mix with gDNA remover (Toyobo).

The expression levels of five immune-related genes were analyzed by quantitative real-time RT-PCR (qRTPCR) assay. Based on the GenBank sequences of the CC chemokine gene (GenBank accession number BB997690), the major histocompatibility complex $(\mathrm{MHC})$ class // $\alpha$ antigen gene (GenBank accession number BB997597), the IgM gene (GenBank accession number BB9976453), the interleukin $1 \beta$ (IL-1 $\beta$ ) gene (GenBank accession number BB997494), the $T$ cell receptor (TCR) a chain gene (GenBank accession number BB997438), and the $\beta$-actin gene (GenBank accession number AB179839), primers were designed using Primer Express version 3.0 software (Applied Biosystems). The primer sequences were as follows: forward primer for the CC chemokine gene, 5'GGACAGTCCTGAAGTGCTTTGA-3'; reverse primer for the CC chemokine gene, 5'-GGTTTGATGCAATGCCATTT-3'; forward primer for the MHC class II $\alpha$ antigen gene, 5'-CCGGTATTGGACCTGCAGTT-3'; reverse primer for the $M H C$ class // $\alpha$ antigen gene, $5^{\prime}$ CTGCACTCGTTCCCTTTGATG-3'; forward primer for the IgM gene, 5'-TACGGCCGGTTTTGACTACTG-3'; reverse primer for the $\lg M$ gene, $5^{\prime}$-AGAACTACATGGCCTCAGAGGAA-3'; forward primer for the $I L-1 \beta$ gene, 5'-TGAAATGCAACTTGAGCGAGAT-3'; reverse primer for the $I L-1 \beta$ gene, 5 '-TGTCATTGGATGATGGGAGATC3'; forward primer for the TCR $\alpha$ chain gene, 5'GTTGAAACCAGGGAGGATTACG-3'; reverse primer for the TCR $\alpha$ chain gene, 5'-TGAGTGCGTTGTATCTGCTGAA-3'; forward primer for the $\beta$-actin gene, $5^{\prime}$ GCCCCACCAGAGCGTAAATA-3'; and reverse primer for the $\beta$-actin gene, 5 -CATCGTACTCCTGCTTGCTGAT-3'. qRT-PCR was performed in triplicate using THUNDERBIRDSYBR qPCR mix (Toyobo) on an ABI7300 real-time PCR instrument (Applied Biosystems). The relative gene expression level was calculated according to the $2^{-\Delta \Delta \mathrm{Ct}}$ method (Livak and Schmittgen, 2001 ), normalized to $\beta$-actin, and represented as mean $n$-fold change compared with the level in the non-administered group not challenged with $N$. seriolae.
Quantification of the 16 S ribosomal RNA gene of $\mathrm{N}$. seriolae

Brain, gill, heart, kidney, liver, and spleen tissue samples at 9 and 18 days p.i. were homogenized in $200 \mu \mathrm{L}$ of lysis buffer (50 mM Tris- $\mathrm{HCl}, \mathrm{pH} 7.5,1 \mathrm{~mm}$ EDTA, $0.2 \%$ Nonidet-40, $1.0 \%$ Tween 20 , and $0.5 \%$ sodium dodecyl sulfate). Proteinase $\mathrm{K}(2 \mu \mathrm{L}, 20 \mathrm{mg}$ $\mathrm{mL}^{-1}$; TaKaRa) was added to the lysis buffer, incubated at $50^{\circ} \mathrm{C}$ overnight, and inactivated at $98^{\circ} \mathrm{C}$ for $10 \mathrm{~min}$. Lysozyme (5 $\mu \mathrm{L}, 2 \mathrm{mg} \mathrm{mL}^{-1}$; Sigma Aldrich) was added to the lysis buffer, and incubated at $37^{\circ} \mathrm{C}$ for $30 \mathrm{~min}$. Proteinase $\mathrm{K}(2 \mu \mathrm{L})$ was added again, and incubated at $55^{\circ} \mathrm{C}$ for $2 \mathrm{~h}$. Genomic DNA was purified by phenolchloroform extraction and the ethanol precipitation method. The pelleted genomic DNA was resuspended in $500 \mu \mathrm{L}$ of TE buffer.

Genomic DNA (200 ng) was subjected to qPCR assay targeting the 165 ribosomal ( $r$ ) RNA gene of $N$. seriolae. The qPCR primers were designed based on the GenBank sequence of the 16S rRNA gene of $N$. seriolae strain DSM 44129 (GenBank accession number NR041861) as follows: forward primer, 5'-ATGGTTCGGGTTGGAAAGATT-3'; and reverse primer, 5'GCCGTGTCTCAGTCCCAGTG-3'. qPCR was performed as described above. The obtained threshold cycle value was converted to the copy number of the 16S rRNA gene by a standard curve. To generate the standard curve, serial 10-fold dilutions of standard plasmid constructed by inserting the 16S rRNA gene fragment using a Mighty TA cloning kit (TaKaRa) were analyzed by qPCR. The copy number of the standard plasmid was calculated from the DNA concentration according to the plasmid size and Avogadro's number.

Sequence analysis of the GAPDH gene of N. seriolae

A fragment of 1,105 bp including the GAPDH gene of $N$. seriolae was amplified by PCR using Ex Taq polymerase. The primers were designed based on the complete genome sequence of Nocardia farcinica (GenBank accession number AP006618) as follows: forward primer, 5'-GACCTTCGACACCCTCGTT-3'; and reverse primer, 5'-ATGAATCCGCTTGAAAGCTG-3'. The amplified product was purified and sequenced using a Big Dye Terminator cycle sequencing kit (Life Technologies) on a 3,130 genetic analyzer instrument (Applied Biosystems). The obtained nucleotide sequence was analyzed using BioEdit. The amino acid sequence was deduced and compared to the GenBank sequences of E. tarda, Edwardsiella ictaluri, A. hydrophila, V. anguillarum, V. alginolyticus, V. harveyi, Lactococcus garviae, Streptococcus iniae, Streptococcus parauberis, Nocardia otitidiscaviarum, Nocardia brasiliensis, Nocardia cyriacigeorgica, Nocardia nova, N. farcinica, and Mycobacterium sp. (GenBank accession numbers BAE07151, HQ697334, HQ697336, HQ697332, HQ697335, HQ697333, EOT30873, AHY16571, 
GAJ61094, WP029924496, WP014986416, WP014351697, WP025350671, WP011210122, and EPQ48177, respectively).

Prediction of B-cell epitopes of GAPDH of E. tarda

B-cell epitopes of GAPDH of $E$. tarda and $N$. seriolae were predicted using $B C P$ red online server (ELManzalawy et al., 2008a) where criteria were set to have $80 \%$ specificity and 12 amino acids in length. VaxiJen v2.0 online server (Doytchinova and Flower, 2007) was used to analyse the antigenicity of epitopes. The predicted epitopes were checked for their conservation and variability with the Immune Epitope Database conservation analysis tool (Bui et al., 2007).

\section{Statistical analysis}

Statistical analysis was performed using Dunnett's one-way ANOVA. In all analyses, $P<0.01$ was taken to indicate statistical significance.

\section{Results and Discussion}

The cumulative survival of fish in the administered and non-administered groups after immersion challenge is shown in Fig. 1. No mortality was observed in the non-administered group not challenged with $N$. seriolae. The mortality of FKC-administered fish started at 12 days p.i., and lasted for 10 days. The cumulative survival was $6.7 \%$. These observations were consistent with the results of Shimahara et al. (2005). The cumulative survival of pGAPDH-administered fish was $2.2 \%$. All rGAPDH-, FKC- and pGAPDH-, and FKC- and rGAPDH-administered fish were dead at 21, 18, and 28 days p.i., respectively. Colonies of $N$. seriolae were recovered from all dead and surviving fish (data not shown). These results indicated that GAPDH of $E$. tarda has no protective effect against $N$. seriolae infection in yellowtail.

To detect and quantify Nocardia species, other studies used a qPCR assay that targeted the 16S rRNA gene (Alfaresi and Elkosh, 2006; Helal et al., 2008; Baek et al., 2009). In this study, we developed a novel qPCR assay that targets the 16S rRNA gene of $N$. seriolae, and determined the copy number in each tissue of fish in the administered groups and the non-administered group challenged with $N$. seriolae at 9 and 18 days p.i. The specificity of the qPCR assay was confirmed by testing the genomic DNA of bacterial pathogens of yellowtail, such as L. garvieae, Photobacterium damselae subsp. piscicida, and Mycobacterium sp. (data not shown). The standard curve used to calculate the copy number of the $16 S$ rRNA gene had a slope

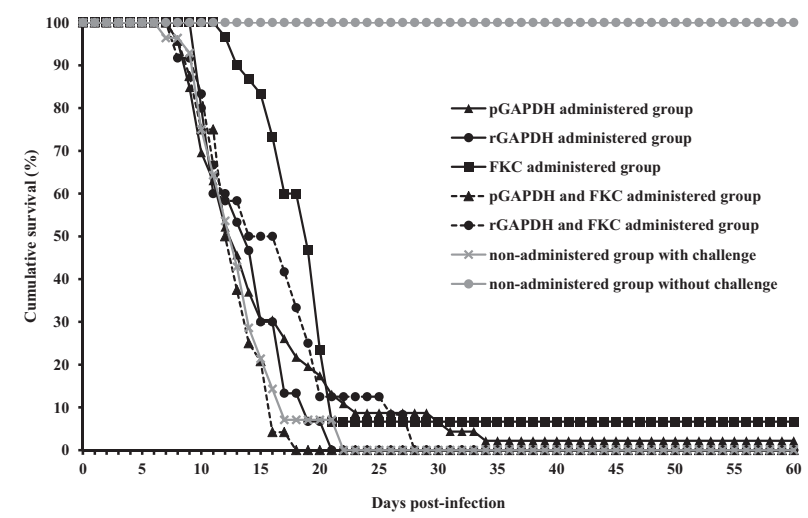

Fig. 1. Cumulative survival of yellowtail in the administered and non-administered groups after immersion challenge with $N$. seriolae.

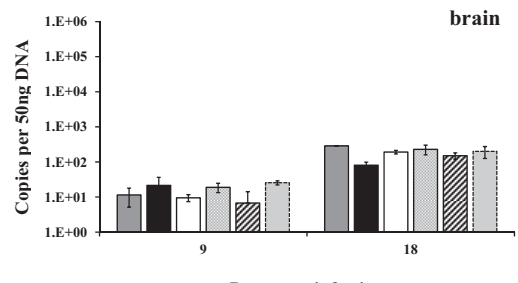

Days post-infection

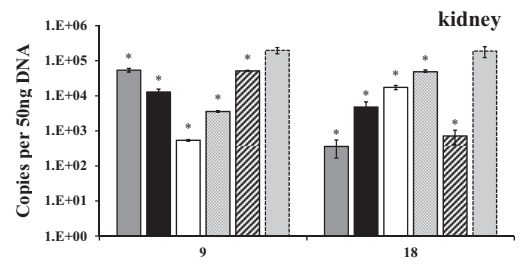

Days post-infection

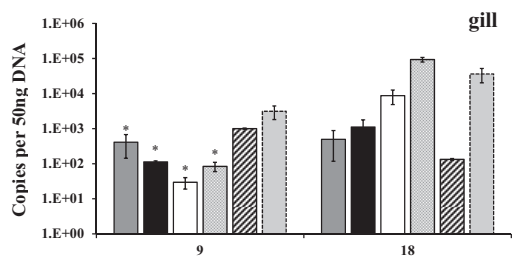

Days post-infection

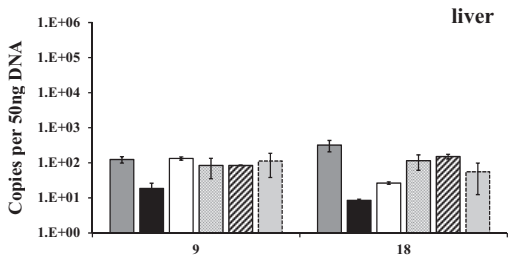

Days post-infection

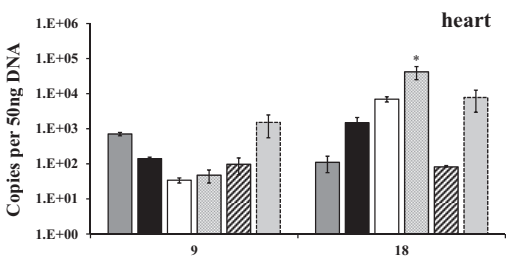

Days post-infection

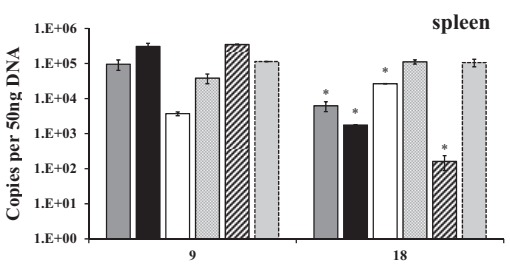

Days post-infection

Fig. 2. Quantitative analysis of the $16 S$ rRNA gene of $N$. seriolae in the brain, gill, heart, kidney, liver, and spleen tissues of yellowtail in the administered groups and the non-administered group challenged with $N$. seriolae. pGAPDH-administered group $\square$; rGAPDH-administered group $\square$; FKC-administered group $\square$; FKC- and pGAPDH-administered group $\square$; FKC and rGAPDH-administered group $\mathbb{Z}$; non-administered group with challenge . Values and error bars represent the means and standard deviation of triplicate measurements. Asterisks indicate statistically significant differences $(P<0.01)$ compared with values of the non-administered group challenged with $N$. seriolae. 
Table 1. Relative expression analysis of five immune-related genes in the liver and spleen of yellowtail in the administered groups and the non-administered group challenged with $N$. seriolae

\begin{tabular}{|c|c|c|c|c|c|c|c|c|c|c|c|}
\hline \multirow{3}{*}{ Group } & \multirow{3}{*}{$\begin{array}{l}\text { Days } \\
\text { p.i. }\end{array}$} & \multicolumn{10}{|c|}{ Gene name } \\
\hline & & \multicolumn{2}{|c|}{ CC chemokine } & \multicolumn{2}{|c|}{$\begin{array}{c}\text { MHC class II } \\
\alpha \text { antigen }\end{array}$} & \multicolumn{2}{|c|}{$\lg M$} & \multicolumn{2}{|c|}{ IL-1 $\beta$} & \multicolumn{2}{|c|}{ TCR $\alpha$ chain } \\
\hline & & Liver & Spleen & Liver & Spleen & Liver & Spleen & Liver & Spleen & Liver & Spleen \\
\hline \multirow{7}{*}{ 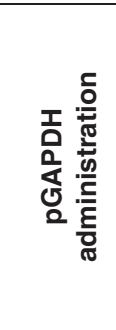 } & 1 & $0.76^{\dagger}$ & $3.15^{*}$ & -0.38 & 1.86 & N.D. & N.D. & 1.18 & 0.66 & -3.92 & 1.61 \\
\hline & 3 & -0.17 & 8.62 & -0.74 & 3.78 & 0.39 & N.D. & N.D. & 1.32 & -2.31 & 1.44 \\
\hline & 5 & -3.16 & 2.01 & -0.36 & -0.57 & N.D. & -1.99 & 1.77 & 2.96 & -1.87 & -3.31 \\
\hline & 7 & N.D. ${ }^{\ddagger}$ & 2.69 & 1.46 & -0.19 & 1.40 & -3.16 & 3.71 & 4.97 & -0.24 & -1.70 \\
\hline & 14 & -4.05 & 3.46 & -2.21 & 1.58 & -1.88 & 0.50 & -1.75 & 2.55 & -3.18 & 1.41 \\
\hline & 36 & 2.47 & 2.46 & 1.09 & 1.64 & 1.97 & 1.84 & 1.92 & -0.90 & -0.25 & 0.44 \\
\hline & 60 & 0.84 & 2.00 & 0.84 & 1.40 & 5.28 & 6.37 & 1.41 & 1.07 & 0.56 & 2.84 \\
\hline \multirow{7}{*}{ 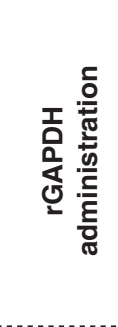 } & 1 & 1.31 & 2.65 & 2.20 & 2.32 & 1.19 & -1.52 & 1.71 & 0.58 & 0.72 & 1.57 \\
\hline & 3 & -2.07 & 9.34 & -1.26 & 2.78 & 0.03 & N.D. & -0.35 & 1.37 & -2.00 & 0.63 \\
\hline & 5 & 1.13 & 4.88 & -0.41 & 3.09 & N.D. & 1.43 & -0.14 & 2.75 & -1.80 & 1.31 \\
\hline & 7 & -1.68 & 3.37 & -0.24 & 1.10 & 0.14 & -1.24 & 3.45 & 5.47 & -2.53 & 0.68 \\
\hline & 14 & -5.31 & 2.25 & -2.69 & 0.21 & -1.77 & 1.30 & -0.32 & 0.07 & -4.04 & -0.53 \\
\hline & 36 & 0.36 & 2.76 & 1.16 & 1.02 & 2.20 & 1.83 & 1.88 & -0.66 & -0.27 & -0.18 \\
\hline & 60 & - & - & - & - & - & - & - & - & - & - \\
\hline \multirow{7}{*}{ 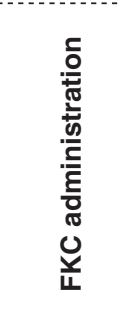 } & 1 & -3.37 & 3.62 & -0.95 & 1.98 & -0.13 & -0.26 & 0.51 & 1.73 & -4.02 & 0.44 \\
\hline & 3 & N.D. & 2.65 & 0.41 & 5.31 & -0.04 & -2.61 & 1.17 & 1.13 & -1.80 & 1.77 \\
\hline & 5 & 0.24 & 2.40 & -0.01 & 2.12 & -0.05 & 0.18 & 2.28 & 3.66 & -0.51 & -0.53 \\
\hline & 7 & -1.01 & 3.79 & -0.64 & 2.50 & -2.47 & 0.11 & 1.95 & 4.49 & -2.31 & 0.68 \\
\hline & 14 & 0.30 & 2.57 & 0.72 & 0.06 & 1.08 & 1.43 & 4.94 & 3.70 & -0.95 & -0.90 \\
\hline & 36 & 2.62 & 1.56 & -1.87 & 1.01 & 2.32 & 2.31 & -2.36 & 1.13 & -2.82 & 1.82 \\
\hline & 60 & 2.43 & 2.27 & 1.60 & 1.49 & 4.42 & 4.52 & 1.29 & 0.29 & 2.33 & 3.03 \\
\hline \multirow{7}{*}{ 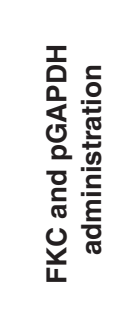 } & 1 & -1.13 & 4.96 & -0.19 & 1.82 & 0.08 & -1.83 & -0.15 & 2.89 & -2.83 & 1.61 \\
\hline & 3 & -2.83 & 2.38 & -0.07 & 1.13 & 0.77 & -0.14 & -1.52 & 0.63 & -3.28 & -0.86 \\
\hline & 5 & -1.46 & 3.43 & -0.45 & 1.62 & -0.80 & -1.55 & 2.50 & 2.44 & -1.73 & 0.53 \\
\hline & 7 & -3.29 & 3.48 & -0.13 & 3.17 & -1.40 & 0.78 & 1.05 & 3.83 & -1.41 & 1.54 \\
\hline & 14 & 0.79 & 3.60 & 0.38 & -1.64 & 0.89 & 1.11 & 3.90 & 3.98 & -2.52 & -2.38 \\
\hline & 36 & 0.24 & 5.13 & -1.60 & 1.64 & 2.53 & 1.92 & -0.95 & 1.21 & -2.17 & 1.39 \\
\hline & 60 & - & - & - & - & - & - & - & - & - & - \\
\hline \multirow{7}{*}{ 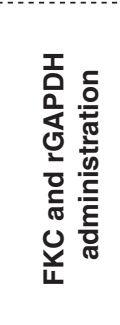 } & 1 & 2.07 & 5.43 & 1.01 & 2.33 & 0.07 & -0.31 & 1.54 & 1.98 & -1.19 & 1.66 \\
\hline & 3 & -1.83 & 2.20 & 0.26 & 2.26 & 1.48 & -1.41 & -0.97 & -0.24 & -3.34 & -1.75 \\
\hline & 5 & -0.07 & 2.69 & 1.23 & 2.53 & 0.88 & -0.85 & 3.96 & 2.47 & -0.16 & 1.41 \\
\hline & 7 & -2.44 & 2.30 & 0.39 & 2.51 & 0.16 & -0.27 & 2.33 & 4.49 & -1.81 & 0.51 \\
\hline & 14 & 0.66 & 3.93 & 0.59 & -0.54 & 0.50 & 0.43 & 4.25 & 1.84 & -1.34 & -1.67 \\
\hline & 36 & 0.61 & 3.52 & 0.74 & 0.83 & 3.99 & 4.12 & 0.60 & 0.38 & -0.72 & -0.77 \\
\hline & 60 & - & - & - & - & - & - & - & - & - & - \\
\hline \multirow{7}{*}{ 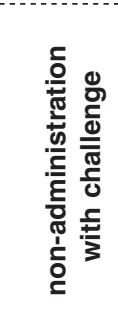 } & 1 & -4.43 & 1.04 & 0.89 & 0.05 & 1.46 & -0.47 & 1.28 & 1.55 & -1.31 & 0.14 \\
\hline & 3 & N.D. & N.D. & -0.01 & 1.13 & -0.35 & N.D. & 1.22 & 1.32 & -1.48 & 1.42 \\
\hline & 5 & N.D. & N.D. & -0.50 & 1.71 & -0.33 & -0.79 & 0.11 & -0.16 & -2.06 & 1.54 \\
\hline & 7 & -2.73 & 1.57 & -1.13 & 1.52 & -2.58 & 0.58 & 0.26 & 5.42 & -3.80 & 0.76 \\
\hline & 14 & 2.10 & 1.62 & 0.20 & -1.43 & 1.30 & 1.04 & 6.53 & 3.13 & -1.89 & -1.63 \\
\hline & 36 & - & - & - & - & - & - & - & - & - & - \\
\hline & 60 & - & - & - & - & - & - & - & - & - & - \\
\hline
\end{tabular}


of -3.427 and a correlation coefficient $R^{2}$ of 0.998 over a range of $2.2-2.2 \times 10^{9}$ copies per reaction (data not shown). As shown in Fig. 2, the copy numbers of the 16S rRNA gene in the gill, kidney, and spleen of fish in each administered group were significantly lower than those in the non-administered group challenged with $N$. seriolae. The lowest copy number was recorded in FKC-administered group at 9 days p.i among the administered groups. In fact, the sampled fish did not have any internal symptoms, while the kidney and spleen of fish of the other administered groups were enlarged with multiple white nodules, which are typical signs of nocardiosis (data not shown). After 9 days p.i., FKCadministered fish also showed the clinical signs.

Microarray analysis to profile immune-related gene expression was developed recently because of poor understanding of the immune system of yellowtail (Darawiroj et al., 2008). The microarray was applied to examine the immune responses elicited by a commer- cial bivalent vaccine for $V$. anguillarum and $L$. garvieae (Nakajima et al., 2012). Of the immune-related genes spotted on the microarray, we chose five genes, and determined genes that were upregulated after administrations with pGAPDH and rGAPDH. Significant upregulation of four immune-related genes other than the TCR $\alpha$ chain gene was detected in each administered group throughout the 60 days of this study compared with those in the non-administered group not challenged with $N$. seriolae (Table 1). It seemed that these immune responses did not differ significantly among the administered groups. As almost all fish in any administered groups were dead, the expression levels of the CC chemokine, MHC class II $\alpha$ antigen, and IL-1 $\beta$ genes were possibly insufficient to overcome $N$. seriolae infection. Alternatively, the three genes may play limited roles in protective immunity against $N$. seriolae. The upregulation of the $\lg M$ gene was likely related to the production of anti- $N$. seriolae antibodies considering
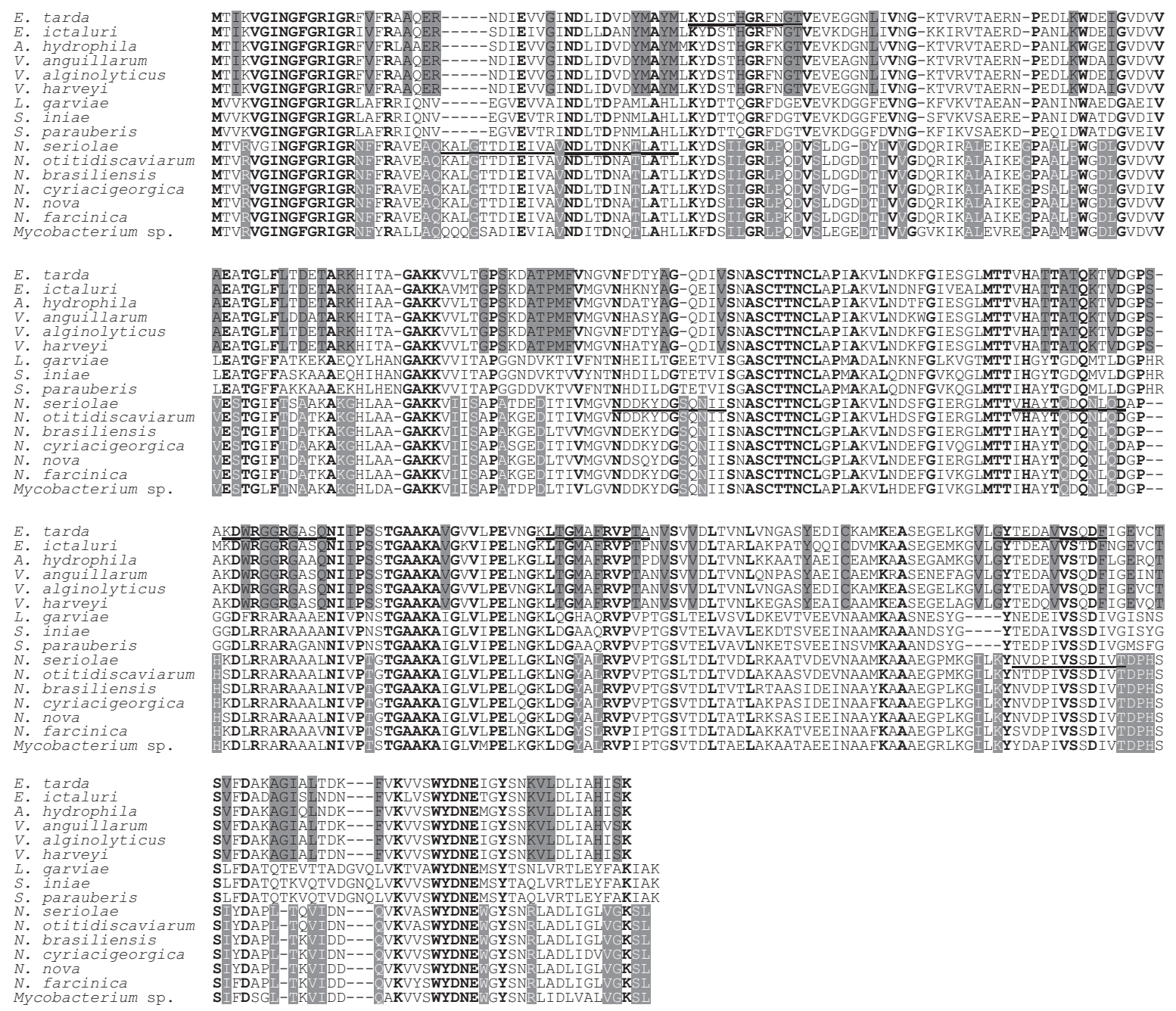

Fig. 3. Comparison of amino acid sequences predicted from the GAPDH gene. Conserved amino acid residues are highlighted in bold. Gray boxes represent amino acid residues conserved only in E. tarda, E. ictaluri, A. hydrophila, V. anguillarum, V. alginolyticus, and $V$. harveyi. Gray boxes with white letters represent amino acid residues conserved only in six Nocardia spp. and Mycobacterium sp. Predicted B-cell epitopes are underlined. 
Kusuda et al. (1989) reported that the agglutination antibody titer against $N$. seriolae significantly increased after administarion with FKC.

Sequence analysis revealed that the GAPDH gene of $N$. seriolae has an amino acid sequence identity of more than $90 \%$ with those of other five Nocardia spp. available in GenBank, while $43 \%$ with that of E. tarda (Fig. 3). Mapping B-cell epitopes is important for vaccine design, immunodiagnostic tests, and antibody production (EL-Manzalawy et al., 2008b). A total of six epitopes of GAPDH of E. tarda, GINGFGRIGRFV (amino acid positions 6 to 17), KYDSTHGRFNGT (amino acid positions 46 to 57), NASCTTNCLAPI (amino acid positions 147 to 158), KDWRGGRGASQN (amino acid positions 192 to 203), KLTGMAFRVPTA (amino acid positions 225 to 236), and GYTEDAVVSQDF (amino acid positions 273 to 284) were predicted using BCPred online server. Antigenic scores were found to be 1.0899 for KYDSTHGRFNGT, 2.2126 for KDWRGGRGASQN, 1.0990 for KLTGMAFRVPTA, and 0.7467 for GYTEDAVVSQDF, respectively, indicating these epitopes as probable antigens. Li et al. (2011) showed that rGAPDHs of E. tarda, E. ictaluri, A. hydrophila, V. anguillarum, V. alginolyticus, and $V$. harveyi shared high levels of identity (over $80 \%$ ) with each other, and gave remarkable cross-protective immunity against those different pathogenic bacteria. Two epitopes, KYDSTHGRFNGT and KDWRGGRGASQN, are completely conserved in $E$. ictaluri and three Vibrio spp., and show approximately $92 \%$ conservation in $A$. hydrophila. KLTGMAFRVPTA or GYTEDAVVSQDF epitope shows approximately $92 \%$ or $75 \%$ conservation in E. ictaluri, approximately $83 \%$ conservation in $A$. hydrophila, and $100 \%$ or approximately $92 \%$ conservation in $V$. harveyi. The two epitopes are completely conserved in $V$. anguillarum and $V$. alginolyticus. These findings suggested that four predicted B-cell epitopes play an important role in cross-protection. This may be a reason that GAPDH of $E$. tarda is not a protective antigen of $N$. seriolae, which was supported by the result that the predicted B-cell epitopes of GAPDH of $N$. seriolae differed from those of E. tarda. Because GAPDHs of members of the family Streptococcaceae i.e., L. garviae, S. iniae, and S. parauberis, or of members of the suborder Corynebacterineae. i.e., N. seriolae, $N$. otitidiscaviarum, $N$. brasiliensis, $N$. cyriacigeorgica, N. nova, N. farcinica, and Mycobacterium sp. share a high amino acid sequence identity, there is a possibility that they have potential for cross-protection. GAPDH of $S$. iniae was identified as immunogenic in olive flounder and Nile tilapia Oreochromis niloticus (Shin et al., 2007; LaFrentz et al., 2011), and therefore its vaccination is expected to cross-protect against fish streptococcosis caused by L. garviae or S. parauberis. Mycobacterium sp. causes mycobacteriosis in cultured yellowtail in Japan, and no effective treatment for this disease is yet available in the aquaculture industry (Kusuda et al., 1987; Kato et al., 2011). We must further examine whether GAPDH of $N$. seriolae protect yellowtail against not only nocardiosis but also mycobacteriosis.

\section{Acknowledgments}

This research was supported by a Grant-in-Aid for Scientific Research from the Japan Society for the Promotion of Science.

\section{References}

Alfaresi, M. and A. Elkosh (2006): Rapid identification of clinically relevant Nocardia species using real-time PCR with SYBR Green and melting-curve analysis. J. Med. Microbiol., 55, 1711-1715.

Baek, K. H., B. D. Yoon, D. H. Cho, B. H. Kim, H. M. Oh and H. S. Kim (2009): Monitoring bacterial population dynamics using real-time PCR during the bioremediation of crude-oilcontaminated soil. J. Microbiol. Biotechnol., 19, 339345 .

Bui, H.-H., J. Sidney, W. Li, N. Fusseder and A. Sette (2007): Development of an epitope conservancy analysis tool to facilitate the design of epitope-based diagnostics and vaccines. BMC Bioinform., 8, 361.

Darawiroj, D., H. Kondo, I. Hirono and T. Aoki (2008): Immunerelated gene expression profiling of yellowtail (Seriola quinqueradiata) kidney cells stimulated with ConA and LPS using microarray analysis. Fish Shellfish Immunol., 24, 260-266.

Doytchinova, I. A. and D. R. Flower (2007): VaxiJen: a server for prediction of protective antigens, tumour antigens and subunit vaccines. BMC Bioinform., 8, 4.

EL-Manzalawy, Y., D. Dobbs and V. Honavar (2008a): Prediction of linear B-cell epitopes using string kernels. J. Mol. Recognit., 21, 243-255.

EL-Manzalawy, Y., D. Dobbs and V. Honavar (2008b): Predicting Protective Linear B-Cell Epitopes Using Evolutionary Information. IEEE International Conference on Bioinformatics and Biomedicine, 289-292.

Helal, Z. H., M. I. Khan, M. S. Ashour and S. A. Eissa (2008): Detection and characterization of Nocardia from patients diagnosed as tuberculosis in Egypt. Int. J. Biomed. Sci., 4, 179-184.

Itano, T., H. Kawakami, T. Kono and M. Sakai (2006): Live vaccine trials against nocardiosis in yellowtail Seriola quinqueradiata. Aquaculture, 261, 1175-1180.

Kato, G., K. Kato, K. Saito, Y. Pe, H. Kondo, T. Aoki and I. Hirono (2011): Vaccine efficacy of Mycobacterium bovis BCG against Mycobacterium sp. infection in amberjack Seriola dumerili. Fish Shellfish Immunol., 30, 467-472.

Kato, G., H. Kondo, T. Aoki and I. Hirono (2012): Mycobacterium bovis $B C G$ vaccine induces non-specific immune responses in Japanese flounder against Nocardia seriolae. Fish Shellfish Immunol., 33, 243-250.

Kawai, K., Y. Liu, K. Ohnishi and S. Oshima (2004): A conserved $37 \mathrm{kDa}$ outer membrane protein of Edwardsiella tarda is an effective vaccine candidate. Vaccine, 22, 3411-3418.

Kudo, K., K. Hatai and A. Seino (1988): Nocardia seriolae sp. nov. Causing nocardiosis of cultured fish. Int. J. Syst. Bacteriol., 38, 173-178. 
Kusuda, R. and A. Nakagawa (1978): Nocardia infection of cultured yellowtail. Fish Pathol., 13, 25-31. (in Japanese with English abstract)

Kusuda, R., K. Kawakami and K. Kawai (1987): A fish-pathogenic Mycobacterium sp. isolated from an epizootic of cultured yellowtail. Nippon Suisan Gakkaishi, 53, 17971804. (in Japanese with English abstract)

Kusuda, R., K. Ono and F. Salati (1988): Changes in agglutinating antibody titers and serum proteins of immunized eels. Dis. Aquat. Organ., 5, 87-92.

Kusuda, R., Y. Kimura and M. Hamaguchi (1989): Changes in peripheral and peritoneal leucocytes in yellowtail Seriola quinqueradiata immunized with Nocardia kampachi. Nippon Suisan Gakkaishi, 55, 1183-1188. (in Japanese with English abstract)

LaFrentz, B. R., C. A. Shoemaker and P. H. Klesius (2011): Immunoproteomic analysis of the antibody response obtained in Nile tilapia following vaccination with a Streptococcus iniae vaccine. Vet. Microbiol., 152, 346-352.

Li, X., H. Wu, M. Zhang, S. Liang, J. Xiao, Q. Wang, Q. Liu and Y. Zhang (2011): Secreted glyceraldehyde-3-phosphate dehydrogenase as a broad spectrum vaccine candidate against microbial infection in aquaculture. Lett. Appl. Microbiol., 54, 1-9.

Liang, S., H. Wu, B. Liu, J. Xiao, Q. Wang and Y. Zhang (2012): Immune response of turbot (Scophthalmus maximus L.) to a broad spectrum vaccine candidate, recombinant glyceraldehyde-3-phosphate dehydrogenase of Edwardsiella tarda. Vet. Immunol. Immunopathol., 150, 198-205.

Liu, Y., S. Oshima, K. Kurohara, K. Ohnishi and K. Kawai (2005): Vaccine efficacy of recombinant GAPDH of Edwardsiella tarda against Edwardsiellosis. Microbiol. Immunol., 49, 605-612.
Liu, Y., S. Oshima and K. Kawai (2007): Glyceraldehyde-3phosphate dehydrogenase of Edwardsiella tarda has protective antigenicity against Vibrio anguillarum in Japanese flounder. Dis. Aquat. Organ., 75, 217-220.

Livak, K. J. and T. D. Schmittgen (2001): Analysis of relative gene expression data using real-time quantitative PCR and the 2(-Delta Delta $\mathrm{C}(\mathrm{T}))$ Method. Methods, 25, 402-408.

Nakajima, N., M. Kijima, M. Kawanishi, G. Katou, K. Futami, T. Katagiri, M. Endo and M. Maita (2012): Analysis of immune response in yellowtail upon vaccination with a bivalent vaccine of Vibrio anguillarum and Lactococcus garvieae. Fish Pathol., 47, 12-19.

Shimahara, Y., H. Yasuda, A. Nakamura, T. Itami and Y. Yoshida (2005): Detection of antibody response against Nocardia seriolae by enzyme-linked immunosorbent assay (ELISA) and a preliminary vaccine trial in yellowtail Seriola quinqueradiata. Bull. Eur. Ass. Fish Pathol., 25, 270-275.

Shimmoto, H., K. Kawai, T. Ikawa and S. Oshima (2010): Protection of red sea bream Pagrus major against red sea bream iridovirus infection by vaccination with a recombinant viral protein. Microbiol. Immunol., 54, 135-142.

Shin, G. W., K. J. Palaksha, Y. R. Kim, S. W. Nho, S. Kim, G. J. Heo, S. C. Park and T. S. Jung (2007): Application of immunoproteomics in developing a Streptococcus iniae vaccine for olive flounder (Paralichthys olivaceus). J. Chromatogr. B: Analyt. Technol. Biomed. Life Sci., 849, 315-322.

Takahashi, Y., K. Fukuda, M. Kondo, S. Yasumoto, I. Hirono and T. Aoki (2011): Bacterial diseases of marine fish and development of vaccine in Japan. J. Nat. Fish. Univ., 60, 51-56. (in Japanese with English abstract) 